\title{
Impulsive quantum difference systems with boundary conditions
}

\author{
Jessada Tariboon ${ }^{1 *}$, Sotiris K Ntouyas ${ }^{2,3}$ and Phollakrit Thiramanus ${ }^{1}$
}

"Correspondence:

jessadat@kmutnb.ac.th

${ }^{1}$ Nonlinear Dynamic Analysis

Research Center, Department of

Mathematics, Faculty of Applied

Science, King Mongkut's University

of Technology North Bangkok,

Bangkok, 10800, Thailand

Full list of author information is

available at the end of the article

\begin{abstract}
This article studies the existence and uniqueness of solutions for coupled systems of nonlinear impulsive quantum difference equations with coupled and uncoupled boundary conditions. The existence and uniqueness of solutions is established by Banach's contraction principle, while the existence of solutions is derived by using Leray-Schauder's alternative. Examples illustrating our results are also presented.
\end{abstract}

MSC: 26A33; 39A13; 34A37

Keywords: quantum calculus; impulsive quantum difference equation; existence; uniqueness; fixed point theorems

\section{Introduction and preliminaries}

In this paper, we concentrate on the study of the existence and uniqueness of solutions for a coupled system of nonlinear impulsive quantum difference equations,

$$
\left\{\begin{array}{l}
D_{q_{k}} x(t)=f(t, x(t), y(t)), \quad t \in J:=[0, T], t \neq t_{k}, \\
D_{p_{k}} y(t)=g(t, x(t), y(t)), \quad t \in J, t \neq t_{k}, \\
\Delta x\left(t_{k}\right)=I_{k}\left(x\left(t_{k}\right)\right), \quad \Delta y\left(t_{k}\right)=I_{k}^{*}\left(y\left(t_{k}\right)\right), \quad k=1,2, \ldots, m, \\
a_{1} x(0)+b_{1} y(T)=\lambda_{1}, \quad a_{2} y(0)+b_{2} x(T)=\lambda_{2},
\end{array}\right.
$$

where $0=t_{0}<t_{1}<t_{2}<\cdots<t_{k}<\cdots<t_{m}<t_{m+1}=T, f, g: J \times \mathbb{R}^{2} \rightarrow \mathbb{R}$ are continuous functions, $I_{k}, I_{k}^{*} \in C(\mathbb{R}, \mathbb{R}), \Delta u\left(t_{k}\right)=u\left(t_{k}^{+}\right)-u\left(t_{k}\right), u\left(t_{k}^{+}\right)=\lim _{h \rightarrow 0^{+}} u\left(t_{k}+h\right), u \in\{x, y\}$, for $k=1,2, \ldots, m$, and $0<p_{k}, q_{k}<1$ for $k=0,1,2, \ldots, m$ are given quantum numbers, $a_{i}, b_{i}, \lambda_{i}$, $i=1,2$ are real constants with $a_{1} a_{2} \neq b_{1} b_{2}$.

The notions of quantum calculus on finite intervals, $q_{k}$-derivatives, and $q_{k}$-integrals were introduced in [1]. For a fixed $k \in \mathbb{N} \cup\{0\}$ let $J_{k}:=\left[t_{k}, t_{k+1}\right] \subset \mathbb{R}$ be an interval and $0<q_{k}<1, k=1,2, \ldots, m$ be a constant. We define the $q_{k}$-derivative of a function $f: J_{k} \rightarrow \mathbb{R}$ at a point $t \in J_{k}$ as follows.

Definition 1.1 Assume $f: J_{k} \rightarrow \mathbb{R}$ is a continuous function and let $t \in J_{k}$. Then the expression

$$
D_{q_{k}} f(t)=\frac{f(t)-f\left(q_{k} t+\left(1-q_{k}\right) t_{k}\right)}{\left(1-q_{k}\right)\left(t-t_{k}\right)}, \quad t \neq t_{k}, \quad D_{q_{k}} f\left(t_{k}\right)=\lim _{t \rightarrow t_{k}} D_{q_{k}} f(t)
$$

is called the $q_{k}$-derivative of function $f$ at $t$.

(c) 2015 Tariboon et al. This article is distributed under the terms of the Creative Commons Attribution 4.0 International License (http://creativecommons.org/licenses/by/4.0/), which permits unrestricted use, distribution, and reproduction in any medium, provided you give appropriate credit to the original author(s) and the source, provide a link to the Creative Commons license, and indicate if changes were made. 
We say that $f$ is $q_{k}$-differentiable on $J_{k}$ provided $D_{q_{k}} f(t)$ exists for all $t \in J_{k}$. Note that if $t_{k}=0$ and $q_{k}=q$ in (1.2), then $D_{q_{k}} f=D_{q} f$, where $D_{q}$ is the well-known $q$-derivative of the function $f(t)$, defined by

$$
D_{q} f(t)=\frac{f(t)-f(q t)}{(1-q) t} .
$$

The $q_{k}$-integral is defined as follows.

Definition 1.2 Assume $f: J_{k} \rightarrow \mathbb{R}$ is a continuous function. Then the $q_{k}$-integral is defined by

$$
\int_{t_{k}}^{t} f(s) d_{q_{k}} s=\left(1-q_{k}\right)\left(t-t_{k}\right) \sum_{n=0}^{\infty} q_{k}^{n} f\left(q_{k}^{n} t+\left(1-q_{k}^{n}\right) t_{k}\right)
$$

for $t \in J_{k}$. Moreover, if $a \in\left(t_{k}, t\right)$, then the definite $q_{k}$-integral is defined by

$$
\begin{aligned}
\int_{a}^{t} f(s) d_{q_{k}} s= & \int_{t_{k}}^{t} f(s) d_{q_{k}} s-\int_{t_{k}}^{a} f(s) d_{q_{k}} s=\left(1-q_{k}\right)\left(t-t_{k}\right) \sum_{n=0}^{\infty} q_{k}^{n} f\left(q_{k}^{n} t+\left(1-q_{k}^{n}\right) t_{k}\right) \\
& -\left(1-q_{k}\right)\left(a-t_{k}\right) \sum_{n=0}^{\infty} q_{k}^{n} f\left(q_{k}^{n} a+\left(1-q_{k}^{n}\right) t_{k}\right) .
\end{aligned}
$$

Note that if $t_{k}=0$ and $q_{k}=q$, then (1.4) reduces to $q$-integral of a function $f(t)$, defined by $\int_{0}^{t} f(s) d_{q} s=(1-q) t \sum_{n=0}^{\infty} q^{n} f\left(q^{n} t\right)$ for $t \in[0, \infty)$.

For the basic properties of the $q_{k}$-derivative and the $q_{k}$-integral we refer to [1].

The book by Kac and Cheung [2] covers many of the fundamental aspects of the quantum calculus. In recent years, the topic of $q$-calculus has attracted the attention of several researchers and a variety of new results can be found in [3-15] and the references cited therein.

Impulsive differential equations serve as basic models to study the dynamics of processes that are subject to sudden changes in their states. The recent development in this field has been motivated by many applied problems, such as control theory, population dynamics, and medicine. For some recent works on the theory of impulsive differential equations, we refer the interested reader to the monographs [16-18]. Moreover, the interested reader is referred to [19-24] for some recent results on impulsive $q_{k}$-difference equations.

In this paper we prove existence and uniqueness results for the impulsive boundary value problem (1.1) by using Banach's contraction mapping principle and Leray-Schauder's nonlinear alternative. The rest of this paper is organized as follows: In Section 2 we present an auxiliary lemma which is used to convert the impulsive boundary value problem (1.1) into an equivalent integral equation. In Section 3, we establish an existence and uniqueness result via Banach's contraction principle, and an existence result by applying LeraySchauder's alternative. Results on uncoupled integral boundary conditions case are in Section 4. Examples illustrating our results are also presented.

\section{An auxiliary lemma}

Let $J=[0, T], J_{0}=\left[t_{0}, t_{1}\right], J_{k}=\left(t_{k}, t_{k+1}\right]$ for $k=1,2, \ldots, m$. To define the solutions of problem (1.1) we need the following lemma, which deals with a linear variant of problem (1.1) and gives a representation of the solutions. 
Tariboon et al. Advances in Difference Equations ( 2015) 2015:163

Page 3 of 19

Lemma 2.1 Given $\phi, \psi \in C(J, \mathbb{R})$, the unique solution of the problem

$$
\left\{\begin{array}{l}
D_{q_{k}} x(t)=\phi(t), \quad t \in J, t \neq t_{k}, \\
D_{p_{k}} y(t)=\psi(t), \quad t \in J, t \neq t_{k}, \\
\Delta x\left(t_{k}\right)=I_{k}\left(x\left(t_{k}\right)\right), \quad \Delta y\left(t_{k}\right)=I_{k}^{*}\left(y\left(t_{k}\right)\right), \quad k=1,2, \ldots, m, \\
a_{1} x(0)+b_{1} y(T)=\lambda_{1}, \quad a_{2} y(0)+b_{2} x(T)=\lambda_{2},
\end{array}\right.
$$

is

$$
\begin{aligned}
x(t)= & \frac{1}{\Omega}\left[a_{2} \lambda_{1}-a_{2} b_{1} \sum_{k=1}^{m}\left(\int_{t_{k-1}}^{t_{k}} \psi(s) d_{p_{k-1}} s+I_{k}^{*}\left(y\left(t_{k}\right)\right)\right)-a_{2} b_{1} \int_{t_{m}}^{T} \psi(s) d_{p_{m}} s\right. \\
& \left.-b_{1} \lambda_{2}+b_{1} b_{2} \sum_{k=1}^{m}\left(\int_{t_{k-1}}^{t_{k}} \phi(s) d_{q_{k-1}} s+I_{k}\left(x\left(t_{k}\right)\right)\right)+b_{1} b_{2} \int_{t_{m}}^{T} \phi(s) d_{q_{m}} s\right] \\
& +\sum_{0<t_{k}<t}\left(\int_{t_{k-1}}^{t_{k}} \phi(s) d_{q_{k-1}} s+I_{k}\left(x\left(t_{k}\right)\right)\right)+\int_{t_{k}}^{t} \phi(s) d_{q_{k}} s
\end{aligned}
$$

and

$$
\begin{aligned}
y(t)= & \frac{1}{\Omega}\left[a_{1} \lambda_{2}-a_{1} b_{2} \sum_{k=1}^{m}\left(\int_{t_{k-1}}^{t_{k}} \phi(s) d_{q_{k-1}} s+I_{k}\left(x\left(t_{k}\right)\right)\right)-a_{1} b_{2} \int_{t_{m}}^{T} \phi(s) d_{q_{m}} s\right. \\
& \left.-b_{2} \lambda_{1}+b_{1} b_{2} \sum_{k=1}^{m}\left(\int_{t_{k-1}}^{t_{k}} \psi(s) d_{p_{k-1}} s+I_{k}^{*}\left(y\left(t_{k}\right)\right)\right)+b_{1} b_{2} \int_{t_{m}}^{T} \psi(s) d_{p_{m}} s\right] \\
& +\sum_{0<t_{k}<t}\left(\int_{t_{k-1}}^{t_{k}} \psi(s) d_{p_{k-1}} s+I_{k}^{*}\left(y\left(t_{k}\right)\right)\right)+\int_{t_{k}}^{t} \psi(s) d_{p_{k}} s,
\end{aligned}
$$

where

$$
\Omega=a_{1} a_{2}-b_{1} b_{2} \neq 0 .
$$

Proof For $t \in J_{0}, q_{0}$-integrating (2.1), it follows that

$$
x(t)=x(0)+\int_{0}^{t} \phi(s) d_{q_{0}} s
$$

which leads to

$$
x\left(t_{1}\right)=x(0)+\int_{0}^{t_{1}} \phi(s) d_{q_{0}} s .
$$

For $t \in J_{1}$, taking the $q_{1}$-integral for (2.1), we get

$$
x(t)=x\left(t_{1}^{+}\right)+\int_{t_{1}}^{t} \phi(s) d_{q_{1}} s
$$

Since $x\left(t_{1}^{+}\right)=x\left(t_{1}\right)+I_{1}\left(x\left(t_{1}\right)\right)$, we have

$$
x(t)=x(0)+\int_{0}^{t_{1}} \phi(s) d_{q_{0}} s+\int_{t_{1}}^{t} \phi(s) d_{q_{1}} s+I_{1}\left(x\left(t_{1}\right)\right) .
$$


Tariboon et al. Advances in Difference Equations ( 2015) 2015:163

Page 4 of 19

Again $q_{2}$-integrating (2.1) from $t_{2}$ to $t$, where $t \in J_{2}$, then

$$
\begin{aligned}
x(t) & =x\left(t_{2}^{+}\right)+\int_{t_{2}}^{t} \phi(s) d_{q_{2}} s \\
& =x(0)+\int_{0}^{t_{1}} \phi(s) d_{q_{0}} s+\int_{t_{1}}^{t_{2}} \phi(s) d_{q_{1}} s+\int_{t_{2}}^{t} \phi(s) d_{q_{2}} s+I_{1}\left(x\left(t_{1}\right)\right)+I_{2}\left(x\left(t_{2}\right)\right) .
\end{aligned}
$$

Repeating the above process, for $t \in J$, we obtain

$$
x(t)=x(0)+\sum_{0<t_{k}<t}\left(\int_{t_{k-1}}^{t_{k}} \phi(s) d_{q_{k-1}} s+I_{k}\left(x\left(t_{k}\right)\right)\right)+\int_{t_{k}}^{t} \phi(s) d_{q_{k}} s .
$$

In the same way, we can obtain

$$
y(t)=y(0)+\sum_{0<t_{k}<t}\left(\int_{t_{k-1}}^{t_{k}} \psi(s) d_{p_{k-1}} s+I_{k}^{*}\left(y\left(t_{k}\right)\right)\right)+\int_{t_{k}}^{t} \psi(s) d_{p_{k}} s .
$$

In particular, for $t=T$, we have

$$
\begin{aligned}
& x(T)=x(0)+\sum_{k=1}^{m}\left(\int_{t_{k-1}}^{t_{k}} \phi(s) d_{q_{k-1}} s+I_{k}\left(x\left(t_{k}\right)\right)\right)+\int_{t_{m}}^{T} \phi(s) d_{q_{m}} s, \\
& y(T)=y(0)+\sum_{k=1}^{m}\left(\int_{t_{k-1}}^{t_{k}} \psi(s) d_{p_{k-1}} s+I_{k}^{*}\left(y\left(t_{k}\right)\right)\right)+\int_{t_{m}}^{T} \psi(s) d_{p_{m}} s .
\end{aligned}
$$

Applying the boundary conditions of (2.1), we get the system

$$
\begin{aligned}
& a_{1} x(0)+b_{1} y(0)+b_{1} \sum_{k=1}^{m}\left(\int_{t_{k-1}}^{t_{k}} \psi(s) d_{p_{k-1}} s+I_{k}^{*}\left(y\left(t_{k}\right)\right)\right)+b_{1} \int_{t_{m}}^{T} \psi(s) d_{p_{m}} s=\lambda_{1}, \\
& a_{2} y(0)+b_{2} x(0)+b_{2} \sum_{k=1}^{m}\left(\int_{t_{k-1}}^{t_{k}} \phi(s) d_{q_{k-1}} s+I_{k}\left(x\left(t_{k}\right)\right)\right)+b_{2} \int_{t_{m}}^{T} \phi(s) d_{q_{m}} s=\lambda_{2},
\end{aligned}
$$

from which we have

$$
\begin{aligned}
x(0)= & \frac{1}{\Omega}\left[a_{2} \lambda_{1}-a_{2} b_{1} \sum_{k=1}^{m}\left(\int_{t_{k-1}}^{t_{k}} \psi(s) d_{p_{k-1}} s+I_{k}^{*}\left(y\left(t_{k}\right)\right)\right)-a_{2} b_{1} \int_{t_{m}}^{T} \psi(s) d_{p_{m}} s\right. \\
& \left.-b_{1} \lambda_{2}+b_{1} b_{2} \sum_{k=1}^{m}\left(\int_{t_{k-1}}^{t_{k}} \phi(s) d_{q_{k-1}} s+I_{k}\left(x\left(t_{k}\right)\right)\right)+b_{1} b_{2} \int_{t_{m}}^{T} \phi(s) d_{q_{m}} s\right]
\end{aligned}
$$

and

$$
\begin{aligned}
y(0)= & \frac{1}{\Omega}\left[a_{1} \lambda_{2}-a_{1} b_{2} \sum_{k=1}^{m}\left(\int_{t_{k-1}}^{t_{k}} \phi(s) d_{q_{k-1}} s+I_{k}\left(x\left(t_{k}\right)\right)\right)-a_{1} b_{2} \int_{t_{m}}^{T} \phi(s) d_{q_{m}} s\right. \\
& \left.-b_{2} \lambda_{1}+b_{1} b_{2} \sum_{k=1}^{m}\left(\int_{t_{k-1}}^{t_{k}} \psi(s) d_{p_{k-1}} s+I_{k}^{*}\left(y\left(t_{k}\right)\right)\right)+b_{1} b_{2} \int_{t_{m}}^{T} \psi(s) d_{p_{m}} s\right] .
\end{aligned}
$$


Substituting the values of $x(0)$ and $y(0)$ in (2.5) and (2.6), we obtain the solutions (2.2) and (2.3).

\section{Main results}

Let $P C(J, \mathbb{R})=\left\{x: J \rightarrow \mathbb{R} ; x(t)\right.$ is continuous everywhere except for some $t_{k}$ at which $x\left(t_{k}^{+}\right)$ and $x\left(t_{k}^{-}\right)$exist and $\left.x\left(t_{k}^{-}\right)=x\left(t_{k}\right), k=1,2, \ldots, m\right\} . P C(J, \mathbb{R})$ is a Banach space with the norm $\|x\|_{P C}=\sup \{|x(t)|, t \in J\}$. Let us introduce the space $X=\{x(t) ; x(t) \in P C([0, T])\}$ endowed with the norm $\|x\|=\sup \{|x(t)|, t \in[0, T]\}$. Obviously $(X,\|\cdot\|)$ is a Banach space. Also let $Y=\{y(t) ; y(t) \in P C([0, T])\}$ be endowed with the norm $\|y\|=\sup \{|y(t)|, t \in[0, T]\}$. Obviously the product space $(X \times Y,\|(x, y)\|)$ is a Banach space with norm $\|(x, y)\|=\|x\|+\|y\|$.

In view of Lemma 2.1, we define an operator $\mathcal{T}: X \times Y \rightarrow X \times Y$ by

$$
\mathcal{T}(x, y)(t)=\left(\begin{array}{l}
\mathcal{T}_{1}(x, y)(t) \\
\mathcal{T}_{2}(x, y)(t)
\end{array}\right)
$$

where

$$
\begin{aligned}
\mathcal{T}_{1}(x, y)(t)= & \frac{1}{\Omega}\left[a_{2} \lambda_{1}-a_{2} b_{1} \sum_{k=1}^{m}\left(\int_{t_{k-1}}^{t_{k}} g(s, x(s), y(s)) d_{p_{k-1}} s+I_{k}^{*}\left(y\left(t_{k}\right)\right)\right)\right. \\
& -a_{2} b_{1} \int_{t_{m}}^{T} g(s, x(s), y(s)) d_{p_{m}} s-b_{1} \lambda_{2} \\
& +b_{1} b_{2} \sum_{k=1}^{m}\left(\int_{t_{k-1}}^{t_{k}} f(s, x(s), y(s)) d_{q_{k-1}} s+I_{k}\left(x\left(t_{k}\right)\right)\right) \\
& \left.+b_{1} b_{2} \int_{t_{m}}^{T} f(s, x(s), y(s)) d_{q_{m}} s\right] \\
& +\sum_{0<t_{k}<t}\left(\int_{t_{k-1}}^{t_{k}} f(s, x(s), y(s)) d_{q_{k-1}} s+I_{k}\left(x\left(t_{k}\right)\right)\right)+\int_{t_{k}}^{t} f(s, x(s), y(s)) d_{q_{k}} s
\end{aligned}
$$

and

$$
\begin{aligned}
\mathcal{T}_{2}(x, y)(t)= & \frac{1}{\Omega}\left[a_{1} \lambda_{2}-a_{1} b_{2} \sum_{k=1}^{m}\left(\int_{t_{k-1}}^{t_{k}} f(s, x(s), y(s)) d_{q_{k-1}} s+I_{k}\left(x\left(t_{k}\right)\right)\right)\right. \\
& -a_{1} b_{2} \int_{t_{m}}^{T} f(s, x(s), y(s)) d_{q_{m}} s-b_{2} \lambda_{1} \\
& +b_{1} b_{2} \sum_{k=1}^{m}\left(\int_{t_{k-1}}^{t_{k}} g(s, x(s), y(s)) d_{p_{k-1}} s+I_{k}^{*}\left(y\left(t_{k}\right)\right)\right) \\
& \left.+b_{1} b_{2} \int_{t_{m}}^{T} g(s, x(s), y(s)) d_{p_{m}} s\right] \\
& +\sum_{0<t_{k}<t}\left(\int_{t_{k-1}}^{t_{k}} g(s, x(s), y(s)) d_{p_{k-1}} s+I_{k}^{*}\left(y\left(t_{k}\right)\right)\right)+\int_{t_{k}}^{t} g(s, x(s), y(s)) d_{p_{k}} s .
\end{aligned}
$$

For the sake of convenience, we set

$$
M_{1}=\frac{1}{|\Omega|}\left[T\left(L_{1}\left|a_{2}\right|\left|b_{1}\right|+K_{1}\left|b_{1}\right|\left|b_{2}\right|+K_{1}|\Omega|\right)+m K_{3}\left(\left|b_{1}\right|\left|b_{2}\right|+|\Omega|\right)\right]
$$




$$
\begin{aligned}
M_{2}= & \frac{1}{|\Omega|}\left[T\left(L_{2}\left|a_{2}\right|\left|b_{1}\right|+K_{2}\left|b_{1}\right|\left|b_{2}\right|+K_{2}|\Omega|\right)+m L_{3}\left|a_{2}\right|\left|b_{1}\right|\right], \\
M_{3}= & \frac{1}{|\Omega|}\left[T\left(N_{2}\left|a_{2}\right|\left|b_{1}\right|+N_{1}\left|b_{1}\right|\left|b_{2}\right|+N_{1}|\Omega|\right)\right. \\
& \left.+m\left(N_{4}\left|a_{2}\right|\left|b_{1}\right|+N_{3}\left|b_{1}\right|\left|b_{2}\right|+N_{3}|\Omega|\right)+\left|a_{2}\right|\left|\lambda_{1}\right|+\left|b_{1}\right|\left|\lambda_{2}\right|\right], \\
M_{4}= & \frac{1}{|\Omega|}\left[T\left(K_{1}\left|a_{1}\right|\left|b_{2}\right|+L_{1}\left|b_{1}\right|\left|b_{2}\right|+L_{1}|\Omega|\right)+m K_{3}\left|a_{1}\right|\left|b_{2}\right|\right], \\
M_{5}= & \frac{1}{|\Omega|}\left[T\left(K_{2}\left|a_{1}\right|\left|b_{2}\right|+L_{2}\left|b_{1}\right|\left|b_{2}\right|+L_{2}|\Omega|\right)+m L_{3}\left(\left|b_{1}\right|\left|b_{2}\right|+|\Omega|\right)\right], \\
M_{6}= & \frac{1}{|\Omega|}\left[T\left(N_{1}\left|a_{1}\right|\left|b_{2}\right|+N_{2}\left|b_{1}\right|\left|b_{2}\right|+N_{2}|\Omega|\right)\right. \\
& \left.+m\left(N_{3}\left|a_{1}\right|\left|b_{2}\right|+N_{4}\left|b_{1}\right|\left|b_{2}\right|+N_{4}|\Omega|\right)+\left|a_{1}\right|\left|\lambda_{2}\right|+\left|b_{2}\right|\left|\lambda_{1}\right|\right] .
\end{aligned}
$$

The first result is concerned with the existence and uniqueness of solutions for the problem (1.1) and is based on Banach's contraction mapping principle.

Theorem 3.1 Assume that:

$\left(\mathrm{H}_{1}\right)$ The functions $f, g:[0, T] \times \mathbb{R}^{2} \rightarrow \mathbb{R}$ are continuous and there exist constants $K_{i}, L_{i}>0$, $i=1,2$ such that for all $t \in[0, T]$ and $u_{i}, v_{i} \in \mathbb{R}, i=1,2$,

$$
\left|f\left(t, u_{1}, u_{2}\right)-f\left(t, v_{1}, v_{2}\right)\right| \leq K_{1}\left|u_{1}-v_{1}\right|+K_{2}\left|u_{2}-v_{2}\right|
$$

and

$$
\left|g\left(t, u_{1}, u_{2}\right)-g\left(t, v_{1}, v_{2}\right)\right| \leq L_{1}\left|u_{1}-v_{1}\right|+L_{2}\left|u_{2}-v_{2}\right| .
$$

$\left(\mathrm{H}_{2}\right)$ The functions $I_{k}, I_{k}^{*}: \mathbb{R} \rightarrow \mathbb{R}$ are continuous and there exist constants $K_{3}, L_{3}>0$ such that for all $t \in[0, T]$ and $u_{3}, v_{3} \in \mathbb{R}, k=1,2, \ldots, m$,

$$
\left|I_{k}\left(u_{3}\right)-I_{k}\left(v_{3}\right)\right| \leq K_{3}\left|u_{3}-v_{3}\right|
$$

and

$$
\left|I_{k}^{*}\left(u_{3}\right)-I_{k}^{*}\left(v_{3}\right)\right| \leq L_{3}\left|u_{3}-v_{3}\right| .
$$

In addition, assume that

$$
M_{1}+M_{2}+M_{4}+M_{5}<1
$$

where $M_{i}, i=1,2,4,5$, are given by (3.1)-(3.2) and (3.4)-(3.5). Then the boundary value problem (1.1) has a unique solution.

Proof Define $\sup _{t \in[0, T]} f(t, 0,0)=N_{1}<\infty, \sup _{t \in[0, T]} g(t, 0,0)=N_{2}<\infty, \sup \left\{\left|I_{k}(0)\right|: k=\right.$ $1,2, \ldots, m\}=N_{3}<\infty$ and $\sup \left\{\left|I_{k}^{*}(0)\right|: k=1,2, \ldots, m\right\}=N_{4}<\infty$ such that

$$
r \geq \max \left\{\frac{M_{3}}{1-\left(M_{1}+M_{2}\right)}, \frac{M_{6}}{1-\left(M_{4}+M_{5}\right)}\right\},
$$

where $M_{3}$ and $M_{6}$ are defined by (3.3) and (3.6), respectively. 
We show that $\mathcal{T} B_{r} \subset B_{r}$, where $B_{r}=\{(x, y) \in X \times Y:\|(x, y)\| \leq r\}$.

For $(x, y) \in B_{r}$, we have

$$
\begin{aligned}
& \left|\mathcal{T}_{1}(x, y)(t)\right| \\
& =\sup _{t \in[0, T]}\left\{\frac { 1 } { \Omega } \left[a_{2} \lambda_{1}-a_{2} b_{1} \sum_{k=1}^{m}\left(\int_{t_{k-1}}^{t_{k}} g(s, x(s), y(s)) d_{p_{k-1}} s+I_{k}^{*}\left(y\left(t_{k}\right)\right)\right)\right.\right. \\
& -a_{2} b_{1} \int_{t_{m}}^{T} g(s, x(s), y(s)) d_{p_{m}} s-b_{1} \lambda_{2} \\
& \left.+b_{1} b_{2} \sum_{k=1}^{m}\left(\int_{t_{k-1}}^{t_{k}} f(s, x(s), y(s)) d_{q_{k-1}} s+I_{k}\left(x\left(t_{k}\right)\right)\right)+b_{1} b_{2} \int_{t_{m}}^{T} f(s, x(s), y(s)) d_{q_{m}} s\right] \\
& \left.+\sum_{0<t_{k}<t}\left(\int_{t_{k-1}}^{t_{k}} f(s, x(s), y(s)) d_{q_{k-1}} s+I_{k}\left(x\left(t_{k}\right)\right)\right)+\int_{t_{k}}^{t} f(s, x(s), y(s)) d_{q_{k}} s\right\} \\
& \leq \frac{1}{|\Omega|}\left[\left|a_{2}\right|\left|\lambda_{1}\right|+\left|a_{2}\right|\left|b_{1}\right| \sum_{k=1}^{m}\left(\int_{t_{k-1}}^{t_{k}}|g(s, x(s), y(s))-g(s, 0,0)|+|g(s, 0,0)| d_{p_{k-1}} s\right.\right. \\
& \left.+\left|I_{k}^{*}\left(y\left(t_{k}\right)\right)-I_{k}^{*}(0)\right|+\left|I_{k}^{*}(0)\right|\right) \\
& +\left|a_{2}\right|\left|b_{1}\right| \int_{t_{m}}^{T}|g(s, x(s), y(s))-g(s, 0,0)|+|g(s, 0,0)| d_{p_{m}} s+\left|b_{1}\right|\left|\lambda_{2}\right| \\
& +\left|b_{1}\right|\left|b_{2}\right| \sum_{k=1}^{m}\left(\int_{t_{k-1}}^{t_{k}}|f(s, x(s), y(s))-f(s, 0,0)|+|f(s, 0,0)| d_{q_{k-1}} s\right. \\
& \left.+\left|I_{k}\left(x\left(t_{k}\right)\right)-I_{k}(0)\right|+\left|I_{k}(0)\right|\right) \\
& \left.+\left|b_{1}\right|\left|b_{2}\right| \int_{t_{m}}^{T}|f(s, x(s), y(s))-f(s, 0,0)|+|f(s, 0,0)| d_{q_{m}} s\right] \\
& +\sum_{k=1}^{m}\left(\int_{t_{k-1}}^{t_{k}}|f(s, x(s), y(s))-f(s, 0,0)|+|f(s, 0,0)| d_{q_{k-1}} s\right. \\
& \left.+\left|I_{k}\left(x\left(t_{k}\right)\right)-I_{k}(0)\right|+\left|I_{k}(0)\right|\right)+\int_{t_{m}}^{t}|f(s, x(s), y(s))-f(s, 0,0)|+|f(s, 0,0)| d_{q_{m}} s \\
& \leq \frac{1}{|\Omega|}\left[\left|a_{2}\right|\left|\lambda_{1}\right|+\left|a_{2}\right|\left|b_{1}\right| \sum_{k=1}^{m}\left(\left(L_{1}\|x\|+L_{2}\|y\|+N_{2}\right)\left(t_{k}-t_{k-1}\right)+L_{3}\|y\|+N_{4}\right)\right. \\
& +\left|a_{2}\right|\left|b_{1}\right|\left(L_{1}\|x\|+L_{2}\|y\|+N_{2}\right)\left(T-t_{m}\right)+\left|b_{1}\right|\left|\lambda_{2}\right| \\
& +\left|b_{1}\right|\left|b_{2}\right| \sum_{k=1}^{m}\left(\left(K_{1}\|x\|+K_{2}\|y\|+N_{1}\right)\left(t_{k}-t_{k-1}\right)+K_{3}\|x\|+N_{3}\right) \\
& \left.+\left|b_{1}\right|\left|b_{2}\right|\left(K_{1}\|x\|+K_{2}\|y\|+N_{1}\right)\left(T-t_{m}\right)\right] \\
& +\sum_{k=1}^{m}\left(\left(K_{1}\|x\|+K_{2}\|y\|+N_{1}\right)\left(t_{k}-t_{k-1}\right)+K_{3}\|x\|+N_{3}\right)
\end{aligned}
$$




$$
\begin{aligned}
& +\left(K_{1}\|x\|+K_{2}\|y\|+N_{1}\right)\left(T-t_{m}\right) \\
= & \frac{1}{|\Omega|}\left[\left|a_{2}\right|\left|\lambda_{1}\right|+\left|a_{2}\right|\left|b_{1}\right| \sum_{k=1}^{m+1}\left(\left(L_{1}\|x\|+L_{2}\|y\|+N_{2}\right)\left(t_{k}-t_{k-1}\right)\right)+\left|a_{2}\right|\left|b_{1}\right| m L_{3}\|y\|\right. \\
& +\left|a_{2}\right|\left|b_{1}\right| m N_{4}+\left|b_{1}\right|\left|\lambda_{2}\right|+\left|b_{1}\right|\left|b_{2}\right| \sum_{k=1}^{m+1}\left(\left(K_{1}\|x\|+K_{2}\|y\|+N_{1}\right)\left(t_{k}-t_{k-1}\right)\right) \\
& \left.+\left|b_{1}\right|\left|b_{2}\right| m K_{3}\|x\|+\left|b_{1}\right|\left|b_{2}\right| m N_{3}\right]+\sum_{k=1}^{m+1}\left(\left(K_{1}\|x\|+K_{2}\|y\|+N_{1}\right)\left(t_{k}-t_{k-1}\right)\right) \\
& +m K_{3}\|x\|+m N_{3} \\
= & \|x\|\left\{\frac{1}{|\Omega|}\left[\sum_{k=1}^{m+1}\left(t_{k}-t_{k-1}\right)\left(L_{1}\left|a_{2}\right|\left|b_{1}\right|+K_{1}\left|b_{1}\right|\left|b_{2}\right|+K_{1}|\Omega|\right)+m K_{3}\left(\left|b_{1}\right|\left|b_{2}\right|+|\Omega|\right)\right]\right\} \\
& +\|y\|\left\{\frac{1}{|\Omega|}\left[\sum_{k=1}^{m+1}\left(t_{k}-t_{k-1}\right)\left(L_{2}\left|a_{2}\right|\left|b_{1}\right|+K_{2}\left|b_{1}\right|\left|b_{2}\right|+K_{2}|\Omega|\right)+m L_{3}\left|a_{2}\right|\left|b_{1}\right|\right]\right\} \\
& +\frac{1}{|\Omega|}\left[\sum_{k=1}^{m+1}\left(t_{k}-t_{k-1}\right)\left(N_{2}\left|a_{2}\right|\left|b_{1}\right|+N_{1}\left|b_{1}\right|\left|b_{2}\right|+N_{1}|\Omega|\right)\right. \\
& \left.+m\left(N_{4}\left|a_{2}\right|\left|b_{1}\right|+N_{3}\left|b_{1}\right|\left|b_{2}\right|+N_{3}|\Omega|\right)+\left|a_{2}\right|\left|\lambda_{1}\right|+\left|b_{1}\right|\left|\lambda_{2}\right|\right] \\
= & M_{1}\|x\|+M_{2}\|y\|+M_{3} \\
\leq & \left(M_{1}+M_{2}\right) r+M_{3} \leq r .
\end{aligned}
$$

In the same way, we can obtain

$$
\begin{aligned}
\left|\mathcal{T}_{2}(x, y)(t)\right| & \\
\leq & \|x\|\left\{\frac{1}{|\Omega|}\left[\sum_{k=1}^{m+1}\left(t_{k}-t_{k-1}\right)\left(K_{1}\left|a_{1}\right|\left|b_{2}\right|+L_{1}\left|b_{1}\right|\left|b_{2}\right|+L_{1}|\Omega|\right)+m K_{3}\left|a_{1}\right|\left|b_{2}\right|\right]\right\} \\
& +\|y\|\left\{\frac { 1 } { | \Omega | } \left[\sum_{k=1}^{m+1}\left(t_{k}-t_{k-1}\right)\left(K_{2}\left|a_{1}\right|\left|b_{2}\right|+L_{2}\left|b_{1}\right|\left|b_{2}\right|+L_{2}|\Omega|\right)\right.\right. \\
& \left.\left.+m L_{3}\left(\left|b_{1}\right|\left|b_{2}\right|+|\Omega|\right)\right]\right\} \\
& +\frac{1}{|\Omega|}\left[\sum_{k=1}^{m+1}\left(t_{k}-t_{k-1}\right)\left(N_{1}\left|a_{1}\right|\left|b_{2}\right|+N_{2}\left|b_{1}\right|\left|b_{2}\right|+N_{2}|\Omega|\right)\right. \\
& \left.+m\left(N_{3}\left|a_{1}\right|\left|b_{2}\right|+N_{4}\left|b_{1}\right|\left|b_{2}\right|+N_{4}|\Omega|\right)+\left|a_{1}\right|\left|\lambda_{2}\right|+\left|b_{2}\right|\left|\lambda_{1}\right|\right] \\
= & M_{4}\|x\|+M_{5}\|y\|+M_{6} \\
\leq & \left(M_{4}+M_{5}\right) r+M_{6} \leq r .
\end{aligned}
$$

Consequently, $\|\mathcal{T}(x, y)(t)\| \leq r$. 
Now for $\left(x_{2}, y_{2}\right),\left(x_{1}, y_{1}\right) \in X \times Y$ and for any $t \in[0, T]$, we get

$$
\begin{aligned}
& \left|\mathcal{T}_{1}\left(x_{2}, y_{2}\right)(t)-\mathcal{T}_{1}\left(x_{1}, y_{1}\right)(t)\right| \\
& \leq \frac{1}{|\Omega|}\left[| a _ { 2 } | | b _ { 1 } | \left(\sum_{k=1}^{m+1} \int_{t_{k-1}}^{t_{k}}\left|g\left(s, x_{2}(s), y_{2}(s)\right)-g\left(s, x_{1}(s), y_{1}(s)\right)\right| d_{p_{k-1}} s\right.\right. \\
& \left.+\sum_{k=1}^{m}\left|I_{k}^{*}\left(y_{2}\left(t_{k}\right)\right)-I_{k}^{*}\left(y_{1}\left(t_{k}\right)\right)\right|\right) \\
& +\left|b_{1}\right|\left|b_{2}\right|\left(\sum_{k=1}^{m+1} \int_{t_{k-1}}^{t_{k}}\left|f\left(s, x_{2}(s), y_{2}(s)\right)-f\left(s, x_{1}(s), y_{1}(s)\right)\right| d_{q_{k-1}} s\right. \\
& \left.\left.+\sum_{k=1}^{m}\left|I_{k}\left(x_{2}\left(t_{k}\right)\right)-I_{k}\left(x_{1}\left(t_{k}\right)\right)\right|\right)\right] \\
& +\sum_{k=1}^{m+1} \int_{t_{k-1}}^{t_{k}}\left|f\left(s, x_{2}(s), y_{2}(s)\right)-f\left(s, x_{1}(s), y_{1}(s)\right)\right| d_{q_{k-1}} s+\sum_{k=1}^{m}\left|I_{k}\left(x_{2}\left(t_{k}\right)\right)-I_{k}\left(x_{1}\left(t_{k}\right)\right)\right| \\
& \leq \frac{1}{|\Omega|}\left[\left|a_{2}\right|\left|b_{1}\right|\left(\sum_{k=1}^{m+1}\left(t_{k}-t_{k-1}\right)\left(L_{1}\left\|x_{2}-x_{1}\right\|+L_{2}\left\|y_{2}-y_{1}\right\|\right)+m L_{3}\left\|y_{2}-y_{1}\right\|\right)\right. \\
& \left.+\left|b_{1}\right|\left|b_{2}\right|\left(\sum_{k=1}^{m+1}\left(t_{k}-t_{k-1}\right)\left(K_{1}\left\|x_{2}-x_{1}\right\|+K_{2}\left\|y_{2}-y_{1}\right\|\right)+m K_{3}\left\|x_{2}-x_{1}\right\|\right)\right] \\
& +\sum_{k=1}^{m+1}\left(t_{k}-t_{k-1}\right)\left(K_{1}\left\|x_{2}-x_{1}\right\|+K_{2}\left\|y_{2}-y_{1}\right\|\right)+m K_{3}\left\|x_{2}-x_{1}\right\| \\
& =\left\|x_{2}-x_{1}\right\|\left\{\frac { 1 } { | \Omega | } \left[\sum_{k=1}^{m+1}\left(t_{k}-t_{k-1}\right)\left(L_{1}\left|a_{2}\right|\left|b_{1}\right|+K_{1}\left|b_{1}\right|\left|b_{2}\right|+K_{1}|\Omega|\right)\right.\right. \\
& \left.\left.+m K_{3}\left(\left|b_{1}\right|\left|b_{2}\right|+|\Omega|\right)\right]\right\} \\
& +\left\|y_{2}-y_{1}\right\|\left\{\frac{1}{|\Omega|}\left[\sum_{k=1}^{m+1}\left(t_{k}-t_{k-1}\right)\left(L_{2}\left|a_{2}\right|\left|b_{1}\right|+K_{2}\left|b_{1}\right|\left|b_{2}\right|+K_{2}|\Omega|\right)+m L_{3}\left|a_{2}\right|\left|b_{1}\right|\right]\right\} \\
& =M_{1}\left\|x_{2}-x_{1}\right\|+M_{2}\left\|y_{2}-y_{1}\right\| \text {, }
\end{aligned}
$$

and consequently we obtain

$$
\left\|\mathcal{T}_{1}\left(x_{2}, y_{2}\right)(t)-\mathcal{T}_{1}\left(x_{1}, y_{1}\right)\right\| \leq\left(M_{1}+M_{2}\right)\left[\left\|x_{2}-x_{1}\right\|+\left\|y_{2}-y_{1}\right\|\right]
$$

Similarly,

$$
\left\|\mathcal{T}_{2}\left(x_{2}, y_{2}\right)(t)-\mathcal{T}_{2}\left(x_{1}, y_{1}\right)\right\| \leq\left(M_{4}+M_{5}\right)\left[\left\|x_{2}-x_{1}\right\|+\left\|y_{2}-y_{1}\right\|\right]
$$

It follows from (3.7) and (3.8) that

$$
\left\|\mathcal{T}\left(x_{2}, y_{2}\right)(t)-\mathcal{T}\left(x_{1}, y_{1}\right)(t)\right\| \leq\left(M_{1}+M_{2}+M_{4}+M_{5}\right)\left[\left\|x_{2}-x_{1}\right\|+\left\|y_{2}-y_{1}\right\|\right]
$$


Since $M_{1}+M_{2}+M_{4}+M_{5}<1$, therefore, $\mathcal{T}$ is a contraction operator. So, by Banach's fixed point theorem, the operator $\mathcal{T}$ has a unique fixed point, which is the unique solution of problem (1.1). This completes the proof.

In the next result, we prove the existence of solutions for problem (1.1) by applying the Leray-Schauder alternative.

For the sake of convenience, we set

$$
\begin{aligned}
M_{7}= & \frac{1}{|\Omega|}\left[T\left(B_{1}\left|a_{2}\right|\left|b_{1}\right|+A_{1}\left|b_{1}\right|\left|b_{2}\right|+A_{1}|\Omega|\right)+m A_{4}\left(\left|b_{1}\right|\left|b_{2}\right|+|\Omega|\right)\right], \\
M_{8}= & \frac{1}{|\Omega|}\left[T\left(B_{2}\left|a_{2}\right|\left|b_{1}\right|+A_{2}\left|b_{1}\right|\left|b_{2}\right|+A_{2}|\Omega|\right)+m B_{4}\left|a_{2}\right|\left|b_{1}\right|\right], \\
M_{9}= & \frac{1}{|\Omega|}\left[T\left(B_{0}\left|a_{2}\right|\left|b_{1}\right|+A_{0}\left|b_{1}\right|\left|b_{2}\right|+A_{0}|\Omega|\right)\right. \\
& \left.+m\left(B_{3}\left|a_{2}\right|\left|b_{1}\right|+A_{3}\left|b_{1}\right|\left|b_{2}\right|+A_{3}|\Omega|\right)+\left|a_{2}\right|\left|\lambda_{1}\right|+\left|b_{1}\right|\left|\lambda_{2}\right|\right], \\
M_{10}= & \frac{1}{|\Omega|}\left[T\left(A_{1}\left|a_{1}\right|\left|b_{2}\right|+B_{1}\left|b_{1}\right|\left|b_{2}\right|+B_{1}|\Omega|\right)+m A_{4}\left|a_{1}\right|\left|b_{2}\right|\right], \\
M_{11}= & \frac{1}{|\Omega|}\left[T\left(A_{2}\left|a_{1}\right|\left|b_{2}\right|+B_{2}\left|b_{1}\right|\left|b_{2}\right|+B_{2}|\Omega|\right)+m B_{4}\left(\left|b_{1}\right|\left|b_{2}\right|+|\Omega|\right)\right], \\
M_{12}= & \frac{1}{|\Omega|}\left[T\left(A_{0}\left|a_{1}\right|\left|b_{2}\right|+B_{0}\left|b_{1}\right|\left|b_{2}\right|+B_{0}|\Omega|\right)\right. \\
& \left.+m\left(A_{3}\left|a_{1}\right|\left|b_{2}\right|+B_{3}\left|b_{1}\right|\left|b_{2}\right|+B_{3}|\Omega|\right)+\left|a_{1}\right|\left|\lambda_{2}\right|+\left|b_{2}\right|\left|\lambda_{1}\right|\right],
\end{aligned}
$$

and

$$
M_{0}=\min \left\{1-\left(M_{7}+M_{10}\right), 1-\left(M_{8}+M_{11}\right)\right\} .
$$

Lemma 3.1 (Leray-Schauder alternative) ([25], p.4) Let $F: E \rightarrow E$ be a completely continuous operator (i.e., a map that is restricted to any bounded set in $E$ is compact). Let

$$
\mathcal{E}(F)=\{x \in E: x=\lambda F(x) \text { for some } 0<\lambda<1\} .
$$

Then either the set $\mathcal{E}(F)$ is unbounded, or $F$ has at least one fixed point.

Theorem 3.2 Assume that:

$\left(\mathrm{H}_{3}\right)$ The functions $f, g:[0, T] \times \mathbb{R}^{2} \rightarrow \mathbb{R}$ are continuous and there exist constants $A_{i}, B_{i} \geq 0$ $(i=1,2)$ and $A_{0}, B_{0}>0$ such that $\forall x_{i} \in \mathbb{R}(i=1,2)$

$$
\left|f\left(t, x_{1}, x_{2}\right)\right| \leq A_{0}+A_{1}\left|x_{1}\right|+A_{2}\left|x_{2}\right|
$$

and

$$
\left|g\left(t, x_{1}, x_{2}\right)\right| \leq B_{0}+B_{1}\left|x_{1}\right|+B_{2}\left|x_{2}\right| .
$$


$\left(\mathrm{H}_{4}\right)$ The functions $I_{k}, I_{k}^{*}: \mathbb{R} \rightarrow \mathbb{R}$ are continuous and there exist constants $A_{4}, B_{4} \geq 0$ and $A_{3}, B_{3}>0$ such that $\forall x_{3} \in \mathbb{R}, k=1,2, \ldots, m$

$$
\left|I_{k}\left(x_{3}\right)\right| \leq A_{3}+A_{4}\left|x_{3}\right|
$$

and

$$
\left|I_{k}^{*}\left(x_{3}\right)\right| \leq B_{3}+B_{4}\left|x_{3}\right|
$$

In addition it is assumed that

$$
M_{7}+M_{10}<1 \text { and } M_{8}+M_{11}<1 \text {, }
$$

where $M_{7}, M_{8}, M_{10}, M_{11}$ are given by (3.9)-(3.10) and (3.12)-(3.13). Then there exists at least one solution for the boundary value problem (1.1).

To prove the theorem we use the following lemma.

Lemma 3.2 Assume that $\left(\mathrm{H}_{3}\right)$ and $\left(\mathrm{H}_{4}\right)$ hold. Then the operator $\mathcal{T}: X \times Y \rightarrow X \times Y$ is completely continuous.

Proof By continuity of functions $f$ and $g$, the operator $\mathcal{T}$ is continuous.

Let $\Theta \subset X \times Y$ be bounded. Then there exist positive constants $P_{1}, P_{2}, P_{3}$, and $P_{4}$ such that

$$
\begin{aligned}
& |f(t, x(t), y(t))| \leq P_{1}, \quad|g(t, x(t), y(t))| \leq P_{2}, \quad \forall(x, y) \in \Theta, \\
& \left|I_{k}(x(t))\right| \leq P_{3}, \quad\left|I_{k}^{*}(y(t))\right| \leq P_{4}, \quad k=1,2, \ldots, m .
\end{aligned}
$$

Then for any $(x, y) \in \Theta$, we have

$$
\begin{aligned}
\left\|\mathcal{T}_{1}(x, y)\right\| & \leq \frac{1}{|\Omega|}\left[\left|a_{2}\right|\left|\lambda_{1}\right|+\left|a_{2}\right|\left|b_{1}\right|\left(\sum_{k=1}^{m+1} \int_{t_{k-1}}^{t_{k}}|g(s, x(s), y(s))| d_{p_{k-1}} s+\sum_{k=1}^{m}\left|I_{k}^{*}\left(y\left(t_{k}\right)\right)\right|\right)\right. \\
& \left.+\left|b_{1}\right|\left|\lambda_{2}\right|+\left|b_{1}\right|\left|b_{2}\right|\left(\sum_{k=1}^{m+1} \int_{t_{k-1}}^{t_{k}}|f(s, x(s), y(s))| d_{q_{k-1}} s+\sum_{k=1}^{m}\left|I_{k}\left(x\left(t_{k}\right)\right)\right|\right)\right] \\
& +\sum_{k=1}^{m+1} \int_{t_{k-1}}^{t_{k}}|f(s, x(s), y(s))| d_{q_{k-1}} s+\sum_{k=1}^{m}\left|I_{k}\left(x\left(t_{k}\right)\right)\right| \\
\leq & \frac{1}{|\Omega|}\left[\left|a_{2}\right|\left|\lambda_{1}\right|+\left|a_{2}\right|\left|b_{1}\right|\left(P_{2} T+m P_{4}\right)+\left|b_{1}\right|\left|\lambda_{2}\right|+\left|b_{1}\right|\left|b_{2}\right|\left(P_{1} T+m P_{3}\right)\right] \\
& +P_{1} T+m P_{3} \\
:= & D_{1} .
\end{aligned}
$$


Similarly, we get

$$
\begin{aligned}
\left\|\mathcal{T}_{2}(x, y)\right\| \leq & \frac{1}{|\Omega|}\left[\left|a_{1}\right|\left|\lambda_{2}\right|+\left|a_{1}\right|\left|b_{2}\right|\left(P_{1} T+m P_{3}\right)+\left|b_{2}\right|\left|\lambda_{1}\right|+\left|b_{1}\right|\left|b_{2}\right|\left(P_{2} T+m P_{4}\right)\right] \\
& +P_{2} T+m P_{4} \\
:= & D_{2} .
\end{aligned}
$$

Thus, it follows from the above inequalities that the operator $\mathcal{T}$ is uniformly bounded.

Next, we show that $\mathcal{T}$ is equicontinuous. Let $v_{1}, v_{2} \in\left(t_{l}, t_{l+1}\right)$ for some $l=0,1, \ldots, m$ with $v_{1}<v_{2}$. Then we have

$$
\begin{aligned}
& \left|\mathcal{T}_{1}\left(x\left(v_{2}\right), y\left(v_{2}\right)\right)-\mathcal{T}_{1}\left(x\left(v_{1}\right), y\left(v_{1}\right)\right)\right| \\
& \quad=\left|\int_{t_{l}}^{v_{2}} f(s, x(s), y(s)) d_{q_{l}} s-\int_{t_{l}}^{v_{1}} f(s, x(s), y(s)) d_{q_{l}}\right| \\
& \quad \leq P_{1}\left|v_{2}-v_{1}\right| .
\end{aligned}
$$

Analogously, we can obtain

$$
\begin{aligned}
& \left|\mathcal{T}_{2}\left(x\left(v_{2}\right), y\left(v_{2}\right)\right)-\mathcal{T}_{2}\left(x\left(v_{1}\right), y\left(v_{1}\right)\right)\right| \\
& \quad=\left|\int_{t_{l}}^{v_{2}} g(s, x(s), y(s)) d_{p_{l}} s-\int_{t_{l}}^{v_{1}} g(s, x(s), y(s)) d_{p_{l}} s\right| \\
& \quad \leq P_{2}\left|v_{2}-v_{1}\right| .
\end{aligned}
$$

Therefore, the operator $\mathcal{T}(x, y)$ is equicontinuous, and thus the operator $\mathcal{T}(x, y)$ is completely continuous.

Proof of Theorem 3.2 By Lemma 3.2 the operator $\mathcal{T}(x, y)$ is completely continuous.

Now, it will be verified that the set $\mathcal{E}=\{(x, y) \in X \times Y \mid(x, y)=\lambda \mathcal{T}(x, y), 0 \leq \lambda \leq 1\}$ is bounded. Let $(x, y) \in \mathcal{E}$, then $(x, y)=\lambda \mathcal{T}(x, y)$. For any $t \in[0, T]$, we have

$$
x(t)=\lambda \mathcal{T}_{1}(x, y)(t), \quad y(t)=\lambda \mathcal{T}_{2}(x, y)(t) .
$$

Then

$$
\begin{aligned}
|x(t)| \leq & \|x\|\left\{\frac { 1 } { | \Omega | } \left[\sum_{k=1}^{m+1}\left(t_{k}-t_{k-1}\right)\left(B_{1}\left|a_{2}\right|\left|b_{1}\right|+A_{1}\left|b_{1}\right|\left|b_{2}\right|+A_{1}|\Omega|\right)\right.\right. \\
& \left.\left.+m A_{4}\left(\left|b_{1}\right|\left|b_{2}\right|+|\Omega|\right)\right]\right\} \\
+ & \|y\|\left\{\frac{1}{|\Omega|}\left[\sum_{k=1}^{m+1}\left(t_{k}-t_{k-1}\right)\left(B_{2}\left|a_{2}\right|\left|b_{1}\right|+A_{2}\left|b_{1}\right|\left|b_{2}\right|+A_{2}|\Omega|\right)+m B_{4}\left|a_{2}\right|\left|b_{1}\right|\right]\right\} \\
& +\frac{1}{|\Omega|}\left[\sum_{k=1}^{m+1}\left(t_{k}-t_{k-1}\right)\left(B_{0}\left|a_{2}\right|\left|b_{1}\right|+A_{0}\left|b_{1}\right|\left|b_{2}\right|+A_{0}|\Omega|\right)\right. \\
& \left.+m\left(B_{3}\left|a_{2}\right|\left|b_{1}\right|+A_{3}\left|b_{1}\right|\left|b_{2}\right|+A_{3}|\Omega|\right)+\left|a_{2}\right|\left|\lambda_{1}\right|+\left|b_{1}\right|\left|\lambda_{2}\right|\right]
\end{aligned}
$$


and

$$
\begin{aligned}
|y(t)| \leq & \|x\|\left\{\frac{1}{|\Omega|}\left[\sum_{k=1}^{m+1}\left(t_{k}-t_{k-1}\right)\left(A_{1}\left|a_{1}\right|\left|b_{2}\right|+B_{1}\left|b_{1}\right|\left|b_{2}\right|+B_{1}|\Omega|\right)+m A_{4}\left|a_{1}\right|\left|b_{2}\right|\right]\right\} \\
& +\|y\|\left\{\frac { 1 } { | \Omega | } \left[\sum_{k=1}^{m+1}\left(t_{k}-t_{k-1}\right)\left(A_{2}\left|a_{1}\right|\left|b_{2}\right|+B_{2}\left|b_{1}\right|\left|b_{2}\right|+B_{2}|\Omega|\right)\right.\right. \\
& \left.\left.+m B_{4}\left(\left|b_{1}\right|\left|b_{2}\right|+|\Omega|\right)\right]\right\} \\
& +\frac{1}{|\Omega|}\left[\sum_{k=1}^{m+1}\left(t_{k}-t_{k-1}\right)\left(A_{0}\left|a_{1}\right|\left|b_{2}\right|+B_{0}\left|b_{1}\right|\left|b_{2}\right|+B_{0}|\Omega|\right)\right. \\
& \left.+m\left(A_{3}\left|a_{1}\right|\left|b_{2}\right|+B_{3}\left|b_{1}\right|\left|b_{2}\right|+B_{3}|\Omega|\right)+\left|a_{1}\right|\left|\lambda_{2}\right|+\left|b_{2}\right|\left|\lambda_{1}\right|\right] .
\end{aligned}
$$

Hence we have

$$
\|x\| \leq M_{7}\|x\|+M_{8}\|y\|+M_{9}
$$

and

$$
\|y\| \leq M_{10}\|x\|+M_{11}\|y\|+M_{12}
$$

which imply that

$$
\|x\|+\|y\| \leq\left(M_{7}+M_{10}\right)\|x\|+\left(M_{8}+M_{11}\right)\|y\|+M_{9}+M_{12} .
$$

Consequently,

$$
\|(x, y)\| \leq \frac{M_{9}+M_{12}}{M_{0}}
$$

for any $t \in[0, T]$, where $M_{0}$ is defined by (3.15), which proves that $\mathcal{E}$ is bounded. Thus, by Lemma 3.1, the operator $\mathcal{T}$ has at least one fixed point. Hence the boundary value problem (1.1) has at least one solution. The proof is complete.

\subsection{Examples}

Example 3.1 Consider the following coupled system of impulsive quantum difference equations with coupled boundary conditions

$$
\left\{\begin{array}{l}
D_{\frac{2 k+1}{k^{2}+k+2}} x(t)=\frac{t \cos ^{2}(\pi t)}{\left(3 e^{t}+4\right)^{2}} \frac{|x(t)|}{|x(t)|+1}+\frac{t+1}{(2 t+4)^{3}} \frac{|y(t)|}{|y(t)|+1}+\frac{3}{2}, \quad t \in[0,2], t \neq t_{k}, \\
D_{\frac{\sqrt{k+1}}{e^{k}+1}} y(t)=\frac{1}{\left(2 t^{t+1}+5\right)^{2}} \sin x(t)+\frac{e^{-2(t+1)}}{7} \cos y(t)+\frac{t^{2}+1}{3}, \quad t \in[0,2], t \neq t_{k}, \\
\Delta x\left(t_{k}\right)=\frac{\left|x\left(t_{k}\right)\right|}{6(k+5)+\left|x\left(t_{k}\right)\right|}, \quad \Delta y\left(t_{k}\right)=\frac{\left|y\left(t_{k}\right)\right|}{7(k+4)+\left|y\left(t_{k}\right)\right|}, \quad t_{k}=\frac{k}{2}, k=1,2,3, \\
2 x(0)+4 y(2)=5, \quad 3 y(0)-2 x(2)=-6 .
\end{array}\right.
$$


Here $q_{k}=(2 k+1) /\left(k^{2}+k+2\right), p_{k}=(\sqrt{k+1}) /\left(e^{k}+1\right), k=0,1,2,3, m=3, T=2, a_{1}=2$, $a_{2}=3, b_{1}=4, b_{2}=-2, \lambda_{1}=5, \lambda_{2}=-6, f(t, x, y)=\left(t \cos ^{2}(\pi t)|x|\right) /\left(\left(\left(3 e^{t}+4\right)^{2}\right)(|x|+1)\right)+((t+$ 1) $|y|) /\left(\left((2 t+4)^{3}\right)(|y|+1)\right)+3 / 2, g(t, x, y)=(\sin x) /\left(2^{t+1}+5\right)^{2}+\left(e^{-2(t+1)} \cos y\right) / 7+\left(t^{2}+1\right) / 3$, $I_{k}(x)=|x| /(6(k+5)+|x|)$, and $I_{k}^{*}(y)=|y| /(7(k+4)+|y|)$. We have $\left|f\left(t, x_{1}, y_{1}\right)-f\left(t, x_{2}, y_{2}\right)\right| \leq$ $\left((2 / 49)\left|x_{1}-x_{2}\right|+(3 / 64)\left|y_{1}-y_{2}\right|\right),\left|g\left(t, x_{1}, y_{1}\right)-g\left(t, x_{2}, y_{2}\right)\right| \leq\left((1 / 49)\left|x_{1}-x_{2}\right|+\left(1 /\left(7 e^{2}\right)\right) \mid y_{1}-\right.$ $\left.y_{2} \mid\right),\left|I_{k}(x)-I_{k}(y)\right| \leq(1 / 36)|x-y|$, and $\left|I_{k}^{*}(x)-I_{k}^{*}(y)\right| \leq(1 / 35)|x-y|$. We can find

$$
\Omega=a_{1} a_{2}-b_{1} b_{2}=14 \neq 0 .
$$

With the given values, it is found that $K_{1}=2 / 49, K_{2}=3 / 64, K_{3}=1 / 36, L_{1}=1 / 49, L_{2}=$ $1 /\left(7 e^{2}\right), L_{3}=1 / 35, M_{1} \simeq 0.29422, M_{2} \simeq 0.25393, M_{4} \simeq 0.11127, M_{5} \simeq 0.22224$, and

$$
M_{1}+M_{2}+M_{4}+M_{5} \simeq 0.88167<1 .
$$

Thus all the conditions of Theorem 3.1 are satisfied. Therefore, by the conclusion of Theorem 3.1, problem (3.16) has a unique solution on [0,2].

Example 3.2 Consider the following coupled system of impulsive quantum difference equations with coupled boundary conditions:

$$
\left\{\begin{array}{l}
D_{\frac{k+1}{\sqrt{k^{2}+e^{k}+1}}} x(t)=\frac{1}{4}+\frac{1}{2(t+5)^{2}} \sin x(t)+\frac{1}{7 \pi^{2}} \tan ^{-1} y(t), \quad t \in[0,3], t \neq t_{k}, \\
D_{\frac{1}{3} \sin \left(\frac{k+1}{10} \pi\right)} y(t)=\frac{t+2}{e}+\frac{1}{40} x(t) \cos y(t)+\frac{1}{2^{t}+45} y(t), \quad t \in[0,3], t \neq t_{k}, \\
\Delta x\left(t_{k}\right)=\frac{1}{4} \tan ^{-1}\left(\frac{x\left(t_{k}\right)}{8}\right)+2, \quad t_{k}=\frac{k}{3}, k=1,2, \ldots, 8, \\
\Delta y\left(t_{k}\right)=\frac{1}{5} \sin \left(\frac{y\left(t_{k}\right)}{6}\right)+3, \quad t_{k}=\frac{k}{3}, k=1,2, \ldots, 8, \\
-x(0)+5 y(3)=-2, \quad 2 y(0)+3 x(3)=5 .
\end{array}\right.
$$

Here $q_{k}=(k+1) /\left(\sqrt{k^{2}+e^{k}+1}\right), p_{k}=(\sin (((k+1) \pi) / 10)) / 3, k=0,1,2, \ldots, 8, m=8, T=3$, $a_{1}=-1, a_{2}=2, b_{1}=5, b_{2}=3, \lambda_{1}=-2, \lambda_{2}=5, f(t, x, y)=(1 / 4)+(\sin x) /\left(2(t+5)^{2}\right)+$ $\left(\tan ^{-1} y\right) /\left(7 \pi^{2}\right), g(t, x, y)=((t+2) / e)+(x \cos y) / 40+(y) /\left(2^{t}+45\right), I_{k}(x)=\left(\tan ^{-1}(x / 8)\right) / 4+2$, and $I_{k}^{*}(y)=(\sin (y / 6)) / 5+3$. We get

$$
\Omega=a_{1} a_{2}-b_{1} b_{2}=-17 \neq 0 .
$$

Since $|f(t, x, y)| \leq A_{0}+A_{1}|x|+A_{2}|y|,|g(t, x, y)| \leq B_{0}+B_{1}|x|+B_{2}|y|$, where $A_{0}=1 / 4, A_{1}=$ $1 / 50, A_{2}=1 /\left(7 \pi^{2}\right), B_{0}=5 / e, B_{1}=1 / 40, B_{2}=1 / 46$, it is found that $M_{7} \simeq 0.62765, M_{8} \simeq$ $0.27696, M_{10} \simeq 0.19588, M_{11} \simeq 0.63239$. Furthermore,

$$
M_{7}+M_{10} \approx 0.82353<1
$$

and

$$
M_{8}+M_{11} \approx 0.90935<1 .
$$

Thus all the conditions of Theorem 3.2 holds true and consequently the conclusion of Theorem 3.2; problem (3.17) has at least one solution on [0,3]. 


\section{Uncoupled boundary conditions case}

In this section, we consider again the system

$$
\left\{\begin{array}{l}
D_{q_{k}} x(t)=f(t, x(t), y(t)), \quad t \in J, t \neq t_{k}, \\
D_{p_{k}} y(t)=g(t, x(t), y(t)), \quad t \in J, t \neq t_{k}, \\
\Delta x\left(t_{k}\right)=I_{k}\left(x\left(t_{k}\right)\right), \quad \Delta y\left(t_{k}\right)=I_{k}^{*}\left(y\left(t_{k}\right)\right), \quad k=1,2, \ldots, m, \\
a_{1} x(0)+b_{1} x(T)=\lambda_{1}, \quad a_{2} y(0)+b_{2} y(T)=\lambda_{2} .
\end{array}\right.
$$

Lemma 4.1 (Auxiliary lemma) For $h \in C([0, T], \mathbb{R})$, the unique solution of the problem

$$
\left\{\begin{array}{l}
D_{q_{k}} x(t)=h(t), \quad t \in J, t \neq t_{k}, \\
\Delta x\left(t_{k}\right)=I_{k}\left(x\left(t_{k}\right)\right), \quad k=1,2, \ldots, m, \\
a_{1} x(0)+b_{1} x(T)=\lambda_{1},
\end{array}\right.
$$

is given by

$$
\begin{aligned}
x(t)= & \frac{\lambda_{1}}{\Lambda}-\frac{b_{1}}{\Lambda}\left(\sum_{k=1}^{m+1} \int_{t_{k-1}}^{t_{k}} h(s) d_{q_{k-1}} s+\sum_{k=1}^{m} I_{k}\left(x\left(t_{k}\right)\right)\right) \\
& +\sum_{0<t_{k}<t}\left(\int_{t_{k-1}}^{t_{k}} h(s) d_{q_{k-1}} s+I_{k}\left(x\left(t_{k}\right)\right)\right)+\int_{t_{k}}^{t} h(s) d_{q_{k}} s,
\end{aligned}
$$

where

$$
\Lambda:=a_{1}+b_{1} \neq 0
$$

In view of Lemma 4.1, we define an operator $\mathfrak{T}: X \times Y \rightarrow X \times Y$ by

$$
\mathfrak{T}(u, v)(t)=\left(\begin{array}{l}
\mathfrak{T}_{1}(u, v)(t) \\
\mathfrak{T}_{2}(u, v)(t)
\end{array}\right),
$$

where

$$
\begin{aligned}
\mathfrak{T}_{1}(u, v)(t)= & \frac{\lambda_{1}}{\Lambda}-\frac{b_{1}}{\Lambda}\left(\sum_{k=1}^{m+1} \int_{t_{k-1}}^{t_{k}} f(s, u(s), v(s)) d_{q_{k-1}} s+\sum_{k=1}^{m} I_{k}\left(u\left(t_{k}\right)\right)\right) \\
& +\sum_{0<t_{k}<t}\left(\int_{t_{k-1}}^{t_{k}} f(s, u(s), v(s)) d_{q_{k-1}} s+I_{k}\left(u\left(t_{k}\right)\right)\right)+\int_{t_{k}}^{t} f(s, u(s), v(s)) d_{q_{k}} s
\end{aligned}
$$

and

$$
\begin{aligned}
\mathfrak{T}_{2}(u, v)(t)= & \frac{\lambda_{2}}{\Phi}-\frac{b_{2}}{\Phi}\left(\sum_{k=1}^{m+1} \int_{t_{k-1}}^{t_{k}} g(s, u(s), v(s)) d_{p_{k-1}} s+\sum_{k=1}^{m} I_{k}^{*}\left(v\left(t_{k}\right)\right)\right) \\
& +\sum_{0<t_{k}<t}\left(\int_{t_{k-1}}^{t_{k}} g(s, u(s), v(s)) d_{p_{k-1}} s+I_{k}^{*}\left(v\left(t_{k}\right)\right)\right) \\
& +\int_{t_{k}}^{t} g(s, u(s), v(s)) d_{p_{k}} s,
\end{aligned}
$$


where

$$
\Phi:=a_{2}+b_{2} \neq 0
$$

We remark that $\mathfrak{T}_{1}$ depends only on $f$ and $\mathfrak{T}_{2}$ only on $g$. We call the above system, for convenience, a 'coupled system with uncoupled boundary conditions'.

In the sequel, we set the constants

$$
\begin{aligned}
& \bar{M}_{1}=\frac{1}{|\Lambda|}\left(T \bar{K}_{1}+m \bar{K}_{3}\right)\left(\left|b_{1}\right|+|\Lambda|\right), \\
& \bar{M}_{2}=\frac{1}{|\Lambda|} T \bar{K}_{2}\left(\left|b_{1}\right|+|\Lambda|\right), \\
& \bar{M}_{3}=\frac{1}{|\Lambda|}\left[\left(T \bar{N}_{1}+m \bar{N}_{3}\right)\left(\left|b_{1}\right|+|\Lambda|\right)+\left|\lambda_{1}\right|\right], \\
& \bar{M}_{4}=\frac{1}{|\Phi|} T \bar{L}_{1}\left(\left|b_{2}\right|+|\Phi|\right), \\
& \bar{M}_{5}=\frac{1}{|\Phi|}\left(T \bar{L}_{2}+m \bar{L}_{3}\right)\left(\left|b_{2}\right|+|\Phi|\right), \\
& \bar{M}_{6}=\frac{1}{|\Phi|}\left[\left(T \bar{N}_{2}+m \bar{N}_{4}\right)\left(\left|b_{2}\right|+|\Phi|\right)+\left|\lambda_{2}\right|\right] .
\end{aligned}
$$

Now we present the existence and uniqueness result for problem (4.1). We do not provide the proof of this result as it is similar to the one for Theorem 3.1.

Theorem 4.1 Assume that:

$\left(\mathrm{H}_{5}\right)$ The functions $f, g:[0, T] \times \mathbb{R}^{2} \rightarrow \mathbb{R}$ are continuous and there exist constants $\bar{K}_{i}, \bar{L}_{i}>0$, $i=1,2$ such that for all $t \in[0, T]$ and $u_{i}, v_{i} \in \mathbb{R}, i=1,2$,

$$
\left|f\left(t, u_{1}, u_{2}\right)-f\left(t, v_{1}, v_{2}\right)\right| \leq \bar{K}_{1}\left|u_{1}-v_{1}\right|+\bar{K}_{2}\left|u_{2}-v_{2}\right|
$$

and

$$
\left|g\left(t, u_{1}, u_{2}\right)-g\left(t, v_{1}, v_{2}\right)\right| \leq \bar{L}_{1}\left|u_{1}-v_{1}\right|+\bar{L}_{2}\left|u_{2}-v_{2}\right| .
$$

$\left(\mathrm{H}_{6}\right)$ The functions $I_{k}, I_{k}^{*}: \mathbb{R} \rightarrow \mathbb{R}$ are continuous and there exist constants $\bar{K}_{3}, \bar{L}_{3}>0$ such that for all $t \in[0, T]$ and $u_{3}, v_{3} \in \mathbb{R}, k=1,2, \ldots, m$

$$
\left|I_{k}\left(u_{3}\right)-I_{k}\left(v_{3}\right)\right| \leq \bar{K}_{3}\left|u_{3}-v_{3}\right|
$$

and

$$
\left|I_{k}^{*}\left(u_{3}\right)-I_{k}^{*}\left(v_{3}\right)\right| \leq \bar{L}_{3}\left|u_{3}-v_{3}\right| .
$$

In addition, assume that

$$
\bar{M}_{1}+\bar{M}_{2}+\bar{M}_{4}+\bar{M}_{5}<1,
$$

where $\bar{M}_{1}, \bar{M}_{2}, \bar{M}_{4}, \bar{M}_{5}$ are given by (4.5)-(4.6) and (4.8)-(4.9), respectively. Then the boundary value problem (4.1) has a unique solution. 
Example 4.1 Consider the following coupled system of impulsive quantum difference equations with uncoupled boundary conditions

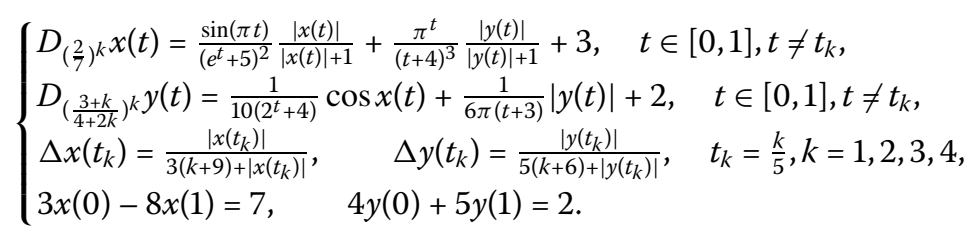

Here $q_{k}=(2 / 7)^{k}, p_{k}=((3+k) /(4+2 k))^{k}, k=0,1,2,3,4, m=4, T=1, a_{1}=3, a_{2}=4, b_{1}=$ $-8, b_{2}=5, \lambda_{1}=7, \lambda_{2}=2, f(t, x, y)=(\sin (\pi t)|x|) /\left(\left(\left(e^{t}+5\right)^{2}\right)(|x|+1)\right)+\left(\pi^{t}|y|\right) /\left(\left((t+4)^{3}\right)(|y|+\right.$ $1))+3, g(t, x, y)=(\cos x) /\left(10\left(2^{t}+4\right)\right)+(|y|) /(6 \pi(t+3))+2, I_{k}(x)=|x| /(3(k+9)+|x|)$, and $I_{k}^{*}(y)=|y| /(5(k+6)+|y|)$. Since $\left|f\left(t, x_{1}, y_{1}\right)-f\left(t, x_{2}, y_{2}\right)\right| \leq\left((1 / 36)\left|x_{1}-x_{2}\right|+(\pi / 64)\left|y_{1}-y_{2}\right|\right)$, $\left|g\left(t, x_{1}, y_{1}\right)-g\left(t, x_{2}, y_{2}\right)\right| \leq\left((1 / 50)\left|x_{1}-x_{2}\right|+(1 /(18 \pi))\left|y_{1}-y_{2}\right|\right),\left|I_{k}(x)-I_{k}(y)\right| \leq(1 / 30)|x-y|$, and $\left|I_{k}^{*}(x)-I_{k}^{*}(y)\right| \leq(1 / 35)|x-y|$. We can find

$$
\Lambda=a_{1}+b_{1}=-5 \neq 0 \text { and } \Phi=a_{2}+b_{2}=9 \neq 0 .
$$

With the given values, it is found that $\bar{K}_{1}=1 / 36, \bar{K}_{2}=\pi / 64, \bar{K}_{3}=1 / 30, \bar{L}_{1}=1 / 50, \bar{L}_{2}=$ $1 /(18 \pi), \bar{L}_{3}=1 / 35, \bar{M}_{1} \simeq 0.41889, \bar{M}_{2} \simeq 0.12763, \bar{M}_{4} \simeq 0.03111, \bar{M}_{5} \simeq 0.20529$, and

$$
\bar{M}_{1}+\bar{M}_{2}+\bar{M}_{4}+\bar{M}_{5} \simeq 0.78291<1 .
$$

Thus all the conditions of Theorem 4.1 are satisfied. Therefore, by the conclusion of Theorem 4.1, problem (4.11) has a unique solution on $[0,1]$.

The second result dealing with the existence of solutions for the problem (4.1) is analogous to Theorem 3.2 and is given below.

In the sequel, we set constants

$$
\begin{aligned}
& \bar{M}_{7}=\frac{1}{|\Lambda|}\left(T \bar{A}_{1}+m \bar{A}_{4}\right)\left(\left|b_{1}\right|+|\Lambda|\right), \\
& \bar{M}_{8}=\frac{1}{|\Lambda|} T \bar{A}_{2}\left(\left|b_{1}\right|+|\Lambda|\right), \\
& \bar{M}_{9}=\frac{1}{|\Lambda|}\left[\left(T \bar{A}_{0}+m \bar{A}_{3}\right)\left(\left|b_{1}\right|+|\Lambda|\right)+\left|\lambda_{1}\right|\right], \\
& \bar{M}_{10}=\frac{1}{|\Phi|} T \bar{B}_{1}\left(\left|b_{2}\right|+|\Phi|\right), \\
& \bar{M}_{11}=\frac{1}{|\Phi|}\left(T \bar{B}_{2}+m \bar{B}_{4}\right)\left(\left|b_{2}\right|+|\Phi|\right), \\
& \bar{M}_{12}=\frac{1}{|\Phi|}\left[\left(T \bar{B}_{0}+m \bar{B}_{3}\right)\left(\left|b_{2}\right|+|\Phi|\right)+\left|\lambda_{2}\right|\right] .
\end{aligned}
$$

\section{Theorem 4.2 Assume that:}

$\left(\mathrm{H}_{7}\right)$ The functions $f, g:[0, T] \times \mathbb{R}^{2} \rightarrow \mathbb{R}$ are continuous and there exist constants $\bar{A}_{i}, \bar{B}_{i} \geq 0$ $(i=1,2)$ and $\bar{A}_{0}, \bar{B}_{0}>0$ such that $\forall x_{i} \in \mathbb{R}(i=1,2)$

$$
\left|f\left(t, x_{1}, x_{2}\right)\right| \leq \bar{A}_{0}+\bar{A}_{1}\left|x_{1}\right|+\bar{A}_{2}\left|x_{2}\right|
$$


and

$$
\left|g\left(t, x_{1}, x_{2}\right)\right| \leq \bar{B}_{0}+\bar{B}_{1}\left|x_{1}\right|+\bar{B}_{2}\left|x_{2}\right|
$$

$\left(\mathrm{H}_{8}\right)$ The functions $I_{k}, I_{k}^{*}: \mathbb{R} \rightarrow \mathbb{R}$ are continuous and there exist constants $\bar{A}_{4}, \bar{B}_{4} \geq 0$ and $\bar{A}_{3}, \bar{B}_{3}>0$ such that $\forall x_{3} \in \mathbb{R}, k=1,2, \ldots, m$,

$$
\left|I_{k}\left(x_{3}\right)\right| \leq \bar{A}_{3}+\bar{A}_{4}\left|x_{3}\right|
$$

and

$$
\left|I_{k}^{*}\left(x_{3}\right)\right| \leq \bar{B}_{3}+\bar{B}_{4}\left|x_{3}\right|
$$

In addition it is assumed that

$$
\bar{M}_{7}+\bar{M}_{10}<1 \text { and } \bar{M}_{8}+\bar{M}_{11}<1
$$

where $\bar{M}_{7}, \bar{M}_{8}, \bar{M}_{10}, \bar{M}_{11}$ are given by (4.12)-(4.13) and (4.15)-(4.16), respectively. Then the boundary value problem (4.1) has at least one solution.

Proof Setting

$$
\bar{M}_{0}=\min \left\{1-\left(\bar{M}_{7}+\bar{M}_{10}\right), 1-\left(\bar{M}_{8}+\bar{M}_{11}\right)\right\},
$$

the proof is similar to that of Theorem 3.2. So we omit it.

Example 4.2 Consider the following coupled system of impulsive quantum difference equations with uncoupled boundary conditions:

$$
\left\{\begin{array}{l}
D_{\frac{2}{3+k}} x(t)=3+\frac{1}{20} \sin \left(\frac{x(t)}{2}\right)+\frac{1}{(t+3)^{2}} \tan ^{-1}\left(\frac{y(t)}{4}\right), \quad t \in[0,1], t \neq t_{k} \\
D_{\frac{3+k}{5+2 k+k^{2}}} y(t)=4+\frac{t}{10\left(2^{t}+1\right)} x(t)+\frac{\sin (\pi t)}{2(2 t+5)^{2}} y(t), \quad t \in[0,1], t \neq t_{k} \\
\Delta x\left(t_{k}\right)=\frac{1}{10 \pi^{2}} \sin \left(\frac{\pi x\left(t_{k}\right)}{2}\right)+\frac{1}{2}, \quad t_{k}=\frac{k}{10}, k=1,2, \ldots, 9 \\
\Delta y\left(t_{k}\right)=\frac{1}{5} \sin \left(\frac{y\left(t_{k}\right)}{6}\right)+3, \quad t_{k}=\frac{k}{10}, k=1,2, \ldots, 9 \\
2 x(0)-7 x(1)=-3, \quad 3 y(0)-5 y(1)=-10 .
\end{array}\right.
$$

Here $q_{k}=2 /(3+k), p_{k}=(3+k) /\left(5+2 k+k^{2}\right), k=0,1,2, \ldots, 9, m=9, T=1, a_{1}=2$, $a_{2}=3, b_{1}=-7, b_{2}=-5, \lambda_{1}=-3, \lambda_{2}=-10, f(t, x, y)=3+(\sin (x / 2)) / 20+\left(\tan ^{-1}(y / 4)\right) /(t+3)^{2}$, $g(t, x, y)=4+(t x) /\left(10\left(2^{t}+1\right)\right)+(\sin (\pi t) y) /\left(2(2 t+5)^{2}\right), I_{k}(x)=(\sin (\pi x / 2)) /\left(10 \pi^{2}\right)+1 / 2$, and $I_{k}^{*}(y)=y /\left(20 e+y^{2}\right)+\pi / 4$. We get

$$
\Lambda=a_{1}+b_{1}=-5 \neq 0 \quad \text { and } \quad \Phi=a_{2}+b_{2}=-2 \neq 0 .
$$

Since $|f(t, x, y)| \leq \bar{A}_{0}+\bar{A}_{1}|x|+\bar{A}_{2}|y|,|g(t, x, y)| \leq \bar{B}_{0}+\bar{B}_{1}|x|+\bar{B}_{2}|y|$, where $\bar{A}_{0}=3, \bar{A}_{1}=1 / 40$, $\bar{A}_{2}=1 / 36, \bar{B}_{0}=4, \bar{B}_{1}=1 / 20, \bar{B}_{2}=1 / 50$, it is found that $\bar{M}_{7} \simeq 0.40377, \bar{M}_{8} \simeq 0.06667$, $\bar{M}_{10} \simeq 0.175, \bar{M}_{11} \simeq 0.64941$. Furthermore,

$$
\bar{M}_{7}+\bar{M}_{10} \approx 0.57877<1
$$


and

$$
\bar{M}_{8}+\bar{M}_{11} \approx 0.71608<1 .
$$

Thus all the conditions of Theorem 4.2 holds true and consequently the conclusion of Theorem 4.2; problem (4.18) has at least one solution on $[0,1]$.

\section{Competing interests}

The authors declare that they have no competing interests.

\section{Authors' contributions}

All authors contributed equally in this article. They read and approved the final manuscript.

\section{Author details}

'Nonlinear Dynamic Analysis Research Center, Department of Mathematics, Faculty of Applied Science, King Mongkut's University of Technology North Bangkok, Bangkok, 10800, Thailand. '2Department of Mathematics, University of loannina, Ioannina, 451 10, Greece. ${ }^{3}$ Nonlinear Analysis and Applied Mathematics (NAAM)-Research Group, Department of Mathematics, Faculty of Science, King Abdulaziz University, P.O. Box 80203, Jeddah, 21589, Saudi Arabia.

\section{Acknowledgements}

We would like to thank the reviewers for their valuable comments and suggestions on the manuscript. This research was funded by King Mongkut's University of Technology North Bangkok. Contract no. KMUTNB-GOV-58-10.

\section{Received: 4 March 2015 Accepted: 17 May 2015 Published online: 02 June 2015}

\section{References}

1. Tariboon, J, Ntouyas, SK: Quantum calculus on finite intervals and applications to impulsive difference equations. Adv. Differ. Equ. 2013, Article ID 282 (2013)

2. Kac, V, Cheung, P: Quantum Calculus. Springer, New York (2002)

3. Bangerezako, G: Variational q-calculus. J. Math. Anal. Appl. 289, 650-665 (2004)

4. Dobrogowska, A, Odzijewicz, A: Second order q-difference equations solvable by factorization method. J. Comput. Appl. Math. 193, 319-346 (2006)

5. Gasper, G, Rahman, M: Some systems of multivariable orthogonal q-Racah polynomials. Ramanujan J. 13, 389-405 (2007)

6. Ismail, MEH, Simeonov, P: q-Difference operators for orthogonal polynomials. J. Comput. Appl. Math. 233, 749-761 (2009)

7. Bohner, M, Guseinov, GS: The h-Laplace and q-Laplace transforms. J. Math. Anal. Appl. 365, 75-92 (2010)

8. El-Shahed, M, Hassan, HA: Positive solutions of q-difference equation. Proc. Am. Math. Soc. 138, 1733-1738 (2010)

9. Ahmad, B: Boundary-value problems for nonlinear third-order q-difference equations. Electron. J. Differ. Equ. 2011, Article ID 94 (2011)

10. Ahmad, B, Alsaedi, A, Ntouyas, SK: A study of second-order q-difference equations with boundary conditions. Adv. Differ. Equ. 2012, Article ID 35 (2012)

11. Ahmad, B, Ntouyas, SK, Purnaras, IK: Existence results for nonlinear q-difference equations with nonlocal boundary conditions. Commun. Appl. Nonlinear Anal. 19, 59-72 (2012)

12. Ahmad, B, Nieto, Jj: On nonlocal boundary value problems of nonlinear q-difference equations. Adv. Differ. Equ. 2012, Article ID 81 (2012)

13. Ahmad, B, Ntouyas, SK: Boundary value problems for q-difference inclusions. Abstr. Appl. Anal. 2011, Article ID $292860(2011)$

14. Zhou, W, Liu, H: Existence solutions for boundary value problem of nonlinear fractional q-difference equations. Adv. Differ. Equ. 2013, Article ID 113 (2013)

15. Yu, C, Wang, J: Existence of solutions for nonlinear second-order $q$-difference equations with first-order q-derivatives. Adv. Differ. Equ. 2013, Article ID 124 (2013)

16. Lakshmikantham, V, Bainov, DD, Simeonov, PS: Theory of Impulsive Differential Equations. World Scientific, Singapore (1989)

17. Samoilenko, AM, Perestyuk, NA: Impulsive Differential Equations. World Scientific, Singapore (1995)

18. Benchohra, M, Henderson, J, Ntouyas, SK: Impulsive Differential Equations and Inclusions, vol. 2. Hindawi Publishing Corporation, New York (2006)

19. Sudsutad, W, Tariboon, J, Ntouyas, SK: Existence of solutions for second-order impulsive q-difference equations with integral boundary conditions. Appl. Math. Inf. Sci. 9, 1147-1157 (2015)

20. Tariboon, J, Ntouyas, SK: Three-point boundary value problems for nonlinear second-order impulsive $q$-difference equations. Adv. Differ. Equ. 2014, Article ID 31 (2014)

21. Tariboon, J, Ntouyas, SK: Boundary value problems for first-order impulsive functional $q$-integro-difference equations. Abstr. Appl. Anal. 2014, Article ID 374565 (2014)

22. Thaiprayoon, C, Tariboon, J, Ntouyas, SK: Separated boundary value problems for second-order impulsive q-integro-difference equations. Adv. Differ. Equ. 2014, Article ID 88 (2014)

23. Asawasamrit, S, Tariboon, J, Ntouyas, SK: Existence of solutions for fractional $q$-integrodifference equations with nonlocal fractional $q$-integral conditions. Abstr. Appl. Anal. 2014, Article ID 474138 (2014)

24. Thiramanus, $P$, Ntouyas, SK, Tariboon, J: Nonlinear second-order impulsive $q$-difference equations. Commun. Appl. Nonlinear Anal. 21(3), 89-102 (2014)

25. Granas, A, Dugundji, J: Fixed Point Theory. Springer, New York (2003) 